Relmecs, junio 2017, vol. 7, no. 1, e017, ISSN 1853-7863

Universidad Nacional de La Plata. Facultad de Humanidades y Ciencias de la Educación. Centro Interdisciplinario de Metodología de las Ciencias Sociales.

Red Latinoamericana de Metodología de las Ciencias Sociales

\title{
Los alcances de un diagnóstico comunitario sobre suicidio: reflexiones a partir de una experiencia
}

\author{
The scope of a community diagnosis on Suicide: Reflections from \\ experience
}

\section{Carolina Castillo Echeverría *, Adriana Maroto Vargas * \\ * Escuela de Sociología, Universidad de Costa Rica, Costa Rica | carolina.castillo e@ucr.ac.cr,} adriana.marotovv@ucr.ac.cr

\section{PALABRAS CLAVE RESUMEN}

Suicidio El artículo analiza los retos y las lecciones aprendidas de un proyecto que realizó un diagnóstico comunitario sobre el suicidio en una comunidad rural en San José, Costa Rica. En un primer momento

Abordaje psicosocial se presenta una reflexión teórica desde el abordaje psicosocial del suicidio y posteriormente los cinco momentos que se siguieron en el proceso (planificación, revisión de información, trabajo de campo,

Diagnóstico comunitario redacción del informe, presentación de resultados y devolución). Se concluye con reflexiones sobre los

Retos retos y las lecciones aprendidas, indicando que el diagnóstico permite conocer la problemática, proponer estrategias de trabajo y facilitar el acercamiento a la comunidad.

Lecciones aprendidas

\section{KEYWORDS ABSTRACT}

Suicide

The article analyzes the challenges and lessons learned from a project that conducted a community assessment of suicide in a rural community in San Jose, Costa Rica. The first section contains a theoretical reflection of suicide from a psychosocial approach and subsequently the five stages that were followed in the process (planning, review of information, field work, report writing and presentation of the results to the community). It concludes with reflections on the challenges and lessons learned, especially how the diagnosis enables understanding the problematic, propose work strategies and facilitate community outreach.

Lessons learned 


\section{Introducción}

A finales del año 2013 ante la solicitud de líderes formales de la comunidad, desde las Escuelas de Sociología y Psicología de la Universidad de Costa Rica, se formuló el proyecto de Acción Social Desarrollo de capacidades en la comunidad de Santa María de Dota para la prevención del suicidio, el cual tuvo como objetivo general contribuir a la comprensión de la problemática del suicidio para desarrollar estrategias de prevención y atención comunitaria de la conducta suicida en esta localidad rural ubicada a unos 60km de San José, Costa Rica.

El cantón de Dota se ubica en la conocida zona de Los Santos -un valle en que se encuentran además los cantones de Tarrazú y León Cortés-, la cual históricamente ha tenido una de las tasas más altas de intento de suicidio y de suicidio consumado en el país.

El proyecto busca abordar la temática del suicidio desde un enfoque psicosocial y comunitario, lo cual ha resultado ser uno de los principales retos debido al enfoque clínico y psicopatologizante que ha predominado sobre el tema, tanto en Costa Rica como a nivel internacional.

La primera actividad propuesta en el marco del proyecto fue realizar un diagnóstico comunitario con el objetivo de identificar tanto aquellos factores que pudieran incidir sobre la conducta suicida en este cantón en particular como las recomendaciones planteadas por las personas de la comunidad sobre cómo abordar la problemática. Desde este enfoque se abordaron las dinámicas comunitarias que pueden actuar como factores protectores y factores de riesgo frente al suicidio, así como también las herramientas que existen en la comunidad tanto para su prevención como para su abordaje.

En este artículo se presenta tanto la metodología utilizada en el proceso anteriormente señalado como las interrogantes que permanecen planteadas. Con esto se busca compartir la experiencia en aras de establecer un intercambio académico con otras personas que estén trabajando desde este enfoque, ya que, como se señaló anteriormente, es un abordaje innovador y es fundamental construir a partir de espacios de encuentro y diálogo. Por lo tanto, cabe aclarar que el objetivo del texto no es la presentación de los resultados de la experiencia, sino una reflexión sobre el proceso, y particularmente sobre la metodología utilizada, lo cual ineludiblemente lleva a algunos apuntes sobre lo teórico para articular la coherencia epistemológica.

\section{El suicidio desde un abordaje psicosocial}

El abordaje psicosocial de los fenómenos sociales necesariamente implica entenderlos como fenómenos complejos en los cuales intervienen factores muy diversos. Es así como una investigación psicosocial sobre el suicidio necesariamente debe interrogar el papel de las instituciones y los grupos de pertenencia de la persona, en tanto estos pueden tener un papel como factores de riesgo o de protección (Carmona, Torbón, Jaramillo y Areiza, 2010). Asimismo, debe entenderse que los diferentes casos de suicidio que se presenten no podrán explicarse desde un único punto de vista o establecerse un perfil unificador de los mismos.

Es también relevante conocer y estudiar las estructuras simbólicas que atribuyen sentido a la realidad, ya que no solo construyen un referente de un fenómeno particular como el suicidio, sino que tienen un peso importante al momento de definir y orientar el comportamiento de las personas respecto al mismo (García, Quintanilla, Sánchez, Morfín y Cruz, 2011).

Investigaciones recientes, como la desarrollada por Chávez, Macías y Luna (2012) en México, afirman que el suicidio se debe reconocer como la manifestación extrema de un dolor psicológico insoportable (en oposición a un trastorno psiquiátrico), y como un evento propiciado por factores psicosociales (en oposición a determinismos biológicos y genéticos). 
Consecuentemente, es importante insistir en la relevancia de conocer los procesos socioculturales implícitos en la construcción e interpretación social del suicidio, ya que es un acto que se manifiesta de manera individual pero que se construye y reconstruye en una intercomunicación social-individual (García Alba et al., 2011).

De tal manera que, a diferencia de como se ha planteado tradicionalmente, la prevención de las conductas suicidas no debería estar exclusivamente centrada en la intervención institucional desde el sector salud sobre las personas y sus factores individuales, sino que requiere de enfoques que tomen en cuenta factores interpersonales, comunitarios y sociales (Suelves y Robert, 2012). Desde esta propuesta de abordaje surgen preguntas tales como: ¿'los procesos de desestructuración del tejido social y los concomitantes efectos de anomia social tienen una relación con la ideación suicida, los intentos suicidas y los suicidios consumados?, ¿la ubicación geográfica de la comunidad puede incidir en la tasa de suicidios debido a la representación simbólica que se hace de la misma?

Asimismo, García Alba et al. (2011) llama la atención respecto a que se debe tener en cuenta que las creencias culturales de los sujetos participan de manera decisiva en la forma en la que enfrentan un problema de salud. Nuevamente surgen interrogantes, ¿el fuerte control social y la imposición de una moral patriarcal y conservadora, especialmente sobre las personas jóvenes, inciden en la tasa de intentos de suicidio y suicidios consumados? En comunidades pequeñas en que hay altas tasas de suicidio, ¿qué papel juegan en la vida cotidiana de la comunidad las historias de las personas que se han suicidado?

Por lo tanto, al ubicar el suicidio desde este abordaje psicosocial se volvió de un interés primordial realizar un diagnóstico que permitiera conocer las diferentes dimensiones de la problemática a partir de la mirada de los actores comunitarios, partiendo del principio de que la población es la que mejor conoce su comunidad, cómo se desarrolla la vida cotidiana y cómo esto puede influir en los suicidios. Son personas que pueden contribuir activamente, no solo con la identificación de sus propios problemas, sino con estrategias que apunten al abordaje y más importante aún, a la prevención del suicidio.

\section{Metodología}

Realizar un diagnóstico comunitario y participativo es un proceso que requiere tiempo. La experiencia que se está reseñando duró 12 meses, aunque se puede realizar en un período menor según los recursos con que se cuente. No obstante, nunca debe perderse de vista que es tan importante el proceso como los resultados finales, así que lo más recomendable es garantizar los recursos necesarios para realizarlo sin presiones administrativas ni logísticas, dejando que sea la dinámica comunitaria misma la que determine su duración.

Hubo dos principios que orientaron todas las actividades que se realizaron: la participación, es decir, la propuesta de que las actividades fueran abiertas e involucraran a personas de diversos sectores de la comunidad, y el diálogo de saberes, entendido a partir de espacios en los que se permitiera la reflexión y el análisis de la problemática identificada desde las diferentes perspectivas.

El proceso tuvo cinco grandes momentos. El primero fue la planificación, la cual empezó con la delimitación teórica del tema -debe buscarse una coherencia epistemológica entre el entendimiento del suicidio y su abordaje- y llegó hasta la propuesta del diagnóstico en el papel. En esta etapa juega un papel importante el conocimiento previo que se tiene de la comunidad. En el caso de la experiencia particular de Dota, se trataba de investigadoras totalmente externas y sin ningún conocimiento previo de la localidad. Esto dificultó imaginar el proceso y sus dificultades ya que no se conocían los actores clave a entrevistar, ni la dinámica comunitaria, y esto, además, se sumaba al problema de ingresar como agentes externos que deben empezar por ganarse la confianza de las personas con las que se va a trabajar. 
En este caso, en el camino se constató que existían experiencias previas en que personas e instituciones habían llegado a trabajar el tema y que, ya fuera por falta de compromiso o por dificultades en las coordinaciones establecidas, no habían logrado cierres adecuados para sus procesos, en los que el denominador común fue la interrupción abrupta y la extracción de la información sin ninguna devolución o trabajo de seguimiento. Por lo tanto, ser un agente externo era un factor a tener muy presente, que hizo necesario contemplar estrategias posibles que minimizaran el impacto negativo que esto pudiera tener. Una de ellas fue la presentación de las investigadoras ante los diferentes actores comunitarios a partir de referencias de otros con los que ya se habían hecho los contactos. La segunda, y quizá la más importante, fue el establecimiento de encuadres claros, así como el seguimiento y cumplimento de los compromisos en los plazos establecidos.

El segundo momento fue la revisión de información específica sobre la comunidad a partir de fuentes secundarias que incluían: (a) historia de la comunidad, (b) estadísticas oficiales de instituciones públicas (tanto a nivel central como local) que remitieran al menos a indicadores sociodemográficos y de salud en general como a los de suicidio en particular, (c) informes sobre proyectos previamente realizados en la comunidad sobre temáticas diversas que estuvieran vinculados al eje de interés. El resultado de este proceso fue un apartado en el informe final que presenta la información de mayor interés, sin embargo, lo más importante es el aporte que significó para las investigadoras el conocimiento de la comunidad más allá de la temática particular de interés.

El tercer momento fue específicamente el trabajo de campo, que se realizó a través de procesos de consulta a personas y grupos de la comunidad. A partir de la experiencia se considera recomendable utilizar al menos las siguientes cuatro técnicas de investigación: las entrevistas, los grupos de discusión y los talleres, y la observación que debe acompañar todo el proceso.

Las entrevistas no estructuradas, tanto individuales como grupales fueron una primera estrategia de acercamiento a la comunidad. El objetivo buscado fue conocer aspectos importantes de la dinámica comunitaria y de las principales problemáticas de salud identificadas, así como tener un primer acercamiento a los factores protectores y de riesgo frente al tema del suicidio. Además, se procuró otro fin -fundamental para el proceso-, que fue el de establecer una relación entre las personas investigadoras y la comunidad.

Madelein (2003) considera que esta técnica -al no ser directiva- permite adoptar un estilo aún más coloquial, de tal manera que permite que la persona entrevistada determine en gran medida el curso de la discusión y que se sienta en un ambiente de confianza. Se realizaron entrevistas tanto individuales como grupales. Estas no se definieron previamente, sino que se aprovecharon las oportunidades que se presentaron mediante la técnica de bola de nieve, según la cual cada persona contactada va brindando información que permite identificar a nuevos informantes claves. En el caso de Dota, se entrevistó a mujeres, representantes de instituciones locales y personas jóvenes, partiendo de la idea de que es importante obtener información de personas con perfiles diversos, ya que así se pueden conocer las perspectivas de diferentes actores sociales.

En Dota, a pesar de que el suicidio está presente en la vida cotidiana, se habla muy poco del tema. Por lo tanto, para facilitar el proceso -y además para concordar con el entendimiento del suicidio desde un enfoque psicosocial- este primer grupo de entrevistas inició con preguntas generales acerca de la salud en la comunidad. Sin contar con una guía estructurada de entrevista, se indagaron aspectos tales como: ¿cómo es la vida en esta comunidad? ¿Cuáles son los principales problemas de la comunidad? ¿Cuáles son los principales problemas de salud y cómo se relacionan con los anteriormente indicados? ¿Cuáles son las necesidades que hay en el área de la salud? Durante la entrevista, el equipo desarrolló preguntas para profundizar o aclarar aspectos señalados por las personas que fueran considerados de interés para el proyecto. 
La atención estuvo puesta en si el tema del suicidio surgía libremente en las conversaciones o si era un tema que se mantenía en silencio. Cuando fue abordado, se hicieron preguntas orientadas a entender cómo la persona explica el suicidio en su comunidad, cuáles factores de riesgo identifica, si considera que hay grupos más vulnerables, entre otros aspectos. En los casos en que el tema no surgió se preguntó directamente al respecto, para conocer si la persona lo consideraba como una problemática o no, sin embargo, estos últimos fueron casos excepcionales.

Estas entrevistas tuvieron duraciones aproximadas entre los 30 y los 90 minutos. Se tomó la decisión de no grabarlas, ya que al ser un primer acercamiento y estar planteadas como una conversación sobre la comunidad en que las personas viven, la grabadora podía resultar amenazante. Por lo tanto, se consideró fundamental que cada entrevista fuera realizada por al menos dos personas, para garantizar tanto la fluidez como el registro de la misma mediante la toma de notas.

El criterio de saturación -entendido como el momento en que las entrevistas no aportan información novedosa o relevante- fue el referente para determinar el fin de esta etapa.

La sistematización fue fundamental en el momento previo al siguiente paso, ya que permitió orientar la recogida de la información mediante las otras técnicas que sí fueron más directivas. Este primer informe permitió identificar y definir la población meta, las temáticas a abordar y el número de sesiones previstas para los grupos focales. En el caso de Dota se tomó la decisión de trabajar con personas jóvenes para abordar factores comunitarios vinculados al suicidio, así como los recursos disponibles en la comunidad; para ello, se programaron dos sesiones de trabajo con cada grupo.

Los grupos focales -según Abarca Rodríguez, Alpízar Rodríguez Sibaja Quesada y Rojas Benavides (2012, p. 161)- buscan generar discusión sobre tópicos muy delimitados.

El término "focal" obedece a que la técnica se limita al estudio de un número reducido de temas; incluso quien modera debe mantener a los participantes circunscritos en los límites del conjunto de temas, por eso quien modera ejerce un papel directivo.

En este caso lo que se buscó someter a discusión fue: (a) los factores de riesgo y los factores protectores vinculados al suicidio identificados en las entrevistas a fin de conocer la valoración que se hace de los mismos, (b) los recursos y las limitaciones que existen en la comunidad para atender los casos que se presentan, y (c) delinear estrategias orientadas a la prevención del suicidio y la promoción de la salud.

Acorde con lo propuesto por Abarca Rodríguez et al. (2014) se siguieron los siguientes pasos para la implementación de los grupos focales: (a) especificación de las características de las personas de interés y los criterios de inclusión/exclusión; (b) selección de los sujetos -se realizaron convocatorias abiertas con el apoyo de los informantes clave e instituciones locales, lo cual no fue muy efectivo-; (c) selección del espacio de reunión en coordinación con una institución local que tenía un espacio físico adecuado; (d) convocatoria a las sesiones con la inclusión espacios agendados para entregar la información, aclarar dudas y extender la invitación; (e) diseño de la guía de preguntas para cada una de las dos sesiones definidas; (f) obtención de datos mediante las sesiones en las que se garantizó que cada persona firmara un asentimiento Informado (menores de edad) y un consentimiento Informado (responsables legales de las personas menores de edad y mayores de edad que participen), además, cada sesión se grabó en audio con la autorización de las personas participantes; finalmente, (g) análisis de la información a partir de la transcripción de cada sesión y la posterior evaluación de los datos mediante la técnica de análisis de contenido.

Se cerró el trabajo de campo con tres talleres de consulta cuyo principal objetivo fue la propuesta de acciones concretas sobre cómo trabajar en la prevención del suicidio en la comunidad. Se consultaron tres grupos: jóvenes, mujeres e instituciones locales. 
En términos generales el taller se trabajó en tres grandes ejes: (a) sondeo para conocer si el suicidio es un tema que se considera importante y si anteriormente se han llevado a cabo iniciativas para abordarlo, (b) árbol de problemas para identificar las causas y consecuencias del suicidio; y (c) árbol de soluciones para abordar algunas de las causas del suicidio que fueron identificadas. En esta última actividad, si bien se dio espacio al "ideal” y a los sueños comunitarios, se hizo un esfuerzo importante por establecer propuestas concretas que se pudieran realizar a corto y mediano plazo con los recursos existentes.

Una de las partes finales fue la sistematización de los resultados de cada uno de estos talleres, con énfasis puesto en la integración de los hallazgos y las propuestas trabajadas en cada uno de ellos.

El cuarto momento fue la redacción del informe final. El informe es claro al explicar tanto el proceso como los resultados, por lo tanto, es un documento que requiere tiempo y debe trabajarse con rigurosidad académica. En el mismo es fundamental garantizar la confidencialidad de las personas que participaron. Es igualmente importante realizar la adaptación de ese informe en algún material que sea adecuado para su distribución en la comunidad, por ejemplo, folletos.

El quinto y último momento fue la presentación y devolución de resultados en la comunidad. Esta etapa fue fundamental, se realizaron al menos cuatro sesiones, incluyendo una entrevista en un medio de comunicación local. El objetivo fue llegar a la mayor cantidad de población posible. En un primer momento se programó una sesión de convocatoria abierta a la cual se invitó a todas las personas que fueron consultadas en el proceso, pero posteriormente se realizaron presentaciones a solicitud de instituciones o grupos organizados.

El proceso de devolución permitió a las personas ver un resultado del diagnóstico (lo cual puede resultar algo abstracto en el momento en que participan en las actividades), lo cual implica ver validadas sus apreciaciones y sus formas de pensar. Es decir, que el diagnóstico no se trata de una lectura de expertos o entes externos que vienen a explicarles sus problemas, sino que éstos se explican desde la lógica de las personas que viven en ese espacio. Además, les permite conocer una propuesta concreta de trabajo y reconocer el compromiso que existe por parte de las personas y la institución que realizaron el diagnóstico, ya que no fue solo un proceso de extracción de información, sino que de manera inmediata se planteó el seguimiento del proyecto.

\section{Retos y lecciones aprendidas en el proceso ${ }^{1}$}

Acorde con los objetivos propuestos para este artículo, no se presentan los resultados de la experiencia que se desarrolló en el cantón de Dota $\underline{\underline{2}}$, sino una discusión sobre la metodología utilizada, las lecciones aprendidas y los retos del proceso.

Martín Zurro y Jodar (2011) afirman que un proyecto que trabaje desde un enfoque de salud comunitaria lo primero que debe plantearse y tener en cuenta es la comunidad particular en que se va a trabajar, aproximarse lo máximo a ella, conocerla y crear alianzas necesarias. Por lo tanto, el diagnóstico comunitario para abordar el tema del suicidio, más que un medio, termina siendo un objetivo propio del proceso.

$\mathrm{Al}$ entender el suicidio desde un enfoque psicosocial y de salud comunitaria se está trascendiendo el enfoque individual, desde el cual se identifican los factores de riesgo y las estrategias de prevención en la persona. Si bien esto es fundamental, especialmente en aquellas personas en que se ha detectado ideación suicida o más aún si han realizado intento suicida, se parte del principio de que en una comunidad con una alta tasa de suicidio debe ampliarse el análisis y el trabajo al marco comunitario. ¿Cómo explicar que en una comunidad haya una alta tasa de suicidio y en otra no? La respuesta a esta pregunta no es sencilla ni se responde en este artículo, pero sí remite a un marco explicativo lo colectivo que de lo individual.

Un proceso orientado desde la salud comunitaria, acorde con la propuesta de Martín Zurro y Jodar (2011), 
debe guiarse por las siguientes preguntas: ¿cuál es el estado de salud de la comunidad? ¿Cuáles son los factores responsables de ese estado de salud? ¿Qué se ha hecho por parte de la comunidad y de los servicios de salud? ¿Qué se puede hacer y cuál sería el impacto esperado de esas acciones? ¿Qué medidas se requieren para dar continuidad a la vigilancia sanitaria de la comunidad y para evaluar los cambios en su estado de salud? A estas interrogantes, para el caso particular que se trabaja en este artículo, se puede añadir: ¿̇se puede entender el suicidio como un síntoma del malestar en la comunidad?

Respecto a la experiencia en Dota, la realización del diagnóstico tuvo dos resultados muy importantes. Por un lado, permitió acceso a la información que se buscaba respecto a los factores que pueden estar incidiendo en el suicidio. Por otro, permitió que las personas de la comunidad conocieran y fueran adquiriendo confianza en las investigadoras. Esto fue fundamental en tanto el diagnóstico estaba planteado como una primera etapa de un proceso a mediano plazo.

En el proceso del diagnóstico surgieron muchas interrogantes que han sido parte muy importante del proceso de aprendizaje. Por esta razón, no solo se presentan sino que también se incluyen algunas líneas de cómo abordarlas según las lecciones aprendidas en el proyecto. No obstante todas ellas responden a situaciones complejas que no tienen una única forma de abordarse.

El primer dilema que se enfrenta -dado que el tema del suicidio suele ser un tabú y hay mucho temor a hablar sobre él- es: ¿el encuadre del proyecto debe plantearse a la comunidad desde un inicio como un proyecto de prevención del suicidio? ¿Cómo van a reaccionar las personas frente a este planteamiento? ¿Estarían dispuestas a participar si se menciona el tema del suicidio? Dota es una comunidad en que el suicidio es parte de la vida cotidiana de una gran parte de la población, ya la mayoría de las personas conocen al menos una historia de intento de suicidio o de suicidio consumado que las ha afectado muy de cerca. Al ser una comunidad pequeña, cada caso suele impactar a varias personas, ya que las mismas comparten todo tipo de relaciones, familiares, en el barrio, en la institución educativa o en el lugar de trabajo, solo por mencionar algunos ejemplos. No obstante, existe un gran silencio que puede extenderse indefinidamente, aun cuando provoque una gran afectación emocional. Se habla desde los rumores debido al impacto que genera la noticia en el momento, pero las personas que son realmente afectadas por esas pérdidas no tienen espacios para hablar sobre el tema, no tienen espacios de escucha de su historia, ni de sus sentimientos, ni de sus pensamientos en relación con la misma.

Por lo tanto, no solo existe temor a enunciar y hablar sobre el tema, sino que hay una gran necesidad de escucha y de sentir que a través de su historia podrían convertirse en la base para construir algún tejido comunitario que pueda brindar mayor soporte a otras personas que estén pasando por situaciones similares.

Las dudas e interrogantes sobre plantear el tema de suicidio de manera directa son válidas. Como no hay seguridad en nombrarlo, se puede partir de la presentación de un proyecto con un enfoque de salud comunitaria (si es que se está planteando desde ese referente). Sin embargo, dado el objetivo de romper con el tabú que existe sobre el tema, es importante no recurrir a estrategias que utilizan otros términos relacionados pero que evitan nombrar el suicidio. Por lo tanto, la recomendación es contribuir a su enunciación y posicionar al suicidio como un tema del que se debe hablar en el espacio público y colectivo.

El segundo dilema que se plantea es: ¿̇a quiénes nos referimos cuando hablamos de la comunidad? Ésta es una discusión muy pertinente desde lo teórico, sin embargo, profundizar en este debate, vigente en disciplinas como la psicología comunitaria, trasciende los objetivos de este trabajo. Por lo tanto, valga acotar que se entiende el concepto de comunidad, acorde con Montero (2004, pp. 207-208), como

Un grupo social histórico, que refleja una cultura preexistente al investigador, que posee cierta organización, cuyos grados varían según el caso, con intereses y necesidades compartidos; que tiene 
su propia vida, en la cual concurre una pluralidad de vidas provenientes de sus miembros; que desarrolla formas de interrelación frecuentes marcadas por la acción, la afectividad, el conocimiento y la información.

Aun con una definición clara, en la práctica, cuando se está planteando un primer acercamiento a la comunidad surgen las siguientes preguntas: ¿con quién hacemos contacto?, ¿cómo gestionamos los contactos?, ¿cómo nos presentamos?, ¿se establecen contactos con personas particulares o grupos organizados?, ¿se debe buscar cierta diversidad en los grupos con los que se trabaja o debe centrarse el trabajo en algunos pocos?, ¿el trabajo con estas personas o grupos es trabajo comunitario? Las respuestas a estas preguntas, nuevamente, no son sencillas y dependen de las particularidades de cada proyecto y de cada comunidad en que se trabaja. Sin embargo, una estrategia que resultó de interés fue hacer contacto con personas clave y trabajar con la técnica de bola de nieve (cada persona sugiere un nuevo contacto acorde a la solicitud que se plantee). En este proceso se establecen contactos, algunos de los cuales resultan estratégicos para el impulso al proyecto, sea porque dan legitimidad a esos agentes externos o porque asumen un compromiso con la iniciativa y van abriendo nuevas posibilidades.

Para el caso del proyecto que funciona como base para estas reflexiones se optó por buscar la participación más amplia y diversa posible -aunque esto respondió a la demanda planteada por la comunidad, la cual dio origen al proyecto-. En otras circunstancias, por falta del tiempo necesario para ello por ejemplo, podría enfocarse el trabajo con grupos más específicos.

El tercer dilema está muy vinculado al punto anterior: en el trabajo van surgiendo preguntas tales como ¿cómo incentivar la participación de las personas en el proyecto? ¿Qué hacer si a las convocatorias grupales llegan dos o tres personas? ¿Evidencia eso que es un tema que no interesa, que es una comunidad poco comprometida con el tema o la iniciativa en particular? Este es un gran desafío que pone en perspectiva los retos de la organización comunitaria, no obstante, la clave está en entender el proyecto como un proceso; conforme este avanza se va logrando mayor reconocimiento de los sectores de la comunidad sobre el trabajo que se está realizando y se van obteniendo mejores resultados. En este proyecto particular la estrategia más efectiva fue el acercamiento a grupos ya organizados. En un inicio se plantearon invitaciones abiertas, que si bien tenían un fin de abrir y democratizar la participación, no tuvieron el poder de convocatoria esperado, lo cual sí fue posible a partir del trabajo con colectivos e instituciones.

Cuarto y último dilema reseñado: ¿Cómo responder frente a la expectativa de la comunidad para la atención de la demanda insatisfecha en lo relativo a los casos de ideación e intento de suicidio, así como de sobrevivientes de suicidio ${ }^{\underline{3}}$ ? En Costa Rica, la atención de la salud mental a través de las instituciones estatales es muy limitada. En el caso del suicidio suele haber una atención a través de los servicios de emergencia y psiquiatría, sin embargo, la atención psicológica (antes, durante y después de la crisis) suele ser mucho menos accesible, razón por la cual existe una alta demanda insatisfecha.

Por lo tanto, un proyecto desde el cual se enuncie el tema, inevitablemente recibirá múltiples solicitudes de atención y seguimiento de casos individuales. Esto debe abordarse de una manera muy ética. En primer lugar, en cada actividad y espacio debe haber un encuadre muy claro sobre los alcances y limitaciones del proyecto a fin de no generar falsas expectativas. En segundo lugar, teniendo lo anterior claro, debe haber una escucha de las situaciones, ya que la intervención en crisis de primer orden o primeros auxilios psicológicos enfatizan la importancia de la escucha en la inmediatez en que la persona lo requiere (Rocamora, 2013). En tercer lugar, debe contribuirse a identificar las instituciones en las que se pueden hacer las referencias, tanto a nivel local como de ciudades cercanas que tengan otra oferta de servicios, tanto públicos como privados.

Como todo trabajo comunitario, las preguntas no hacen más que fortalecer el trabajo realizado, siempre y cuando se busquen espacios para plantearlas y construir alternativas posibles. 


\section{Conclusiones}

En una comunidad con altas tasas de suicidio se debe trascender el abordaje individual y plantear interrogantes sobre qué factores sociales pueden estar incidiendo. Por lo tanto, trabajar el tema del suicidio desde un enfoque comunitario no implica descalificar los abordajes clínicos e individuales, sino reconocer que estos últimos por sí mismos son insuficientes para la atención de la problemática, y que para elaborar una estrategia de prevención es primordial el enfoque de promoción de la salud (mental) sustentado desde los espacios colectivos.

El tema del suicidio suele ser considerado un tabú, tanto en el abordaje de las diferentes profesiones como en la vida cotidiana de las personas y las comunidades. Sin embargo, desde la experiencia particular que se reseñó en este artículo, en esta comunidad en que existen altas tasas de intento de suicidio y suicidio, pero en la cual ha habido un gran silencio y no han habido iniciativas sistemáticas de prevención y atención del mismo, lo que se encuentra es una gran apertura y necesidad de abordar el tema, sea en modalidad de capacitación (cuando se trata de instituciones locales), sea de escucha (cuando se trata de personas que han vivido experiencias), sea de construcción de estrategias (cuando se trata de actores sociales que han colocado el tema como una prioridad en su agenda).

En este sentido, un diagnóstico focalizado a nivel local permite conocer la manera en que las personas explican la problemática en su comunidad e identificar posibles estrategias de trabajo vinculadas a los intereses y los recursos locales, a la vez que permite la presentación de los agentes externos en un proceso gradual que le brinde mayor viabilidad a un proyecto que busca promover las capacidades locales.

\section{Notas}

1 Algunas de estas reflexiones tienen como base el artículo "Los desafíos asociados al uso del enfoque de Salud Comunitaria para comprender y prevenir el suicidio", presentado ante un Comité Editorial de la Escuela de Psicología de la Universidad de Costa Rica para el Libro Miradas sentidas y situadas: experiencias con grupos y comunidades, el cual está siendo sometido a consideración de la Editorial de la Universidad de Costa Rica.

$\underline{2}$ En el caso de la comunidad de Dota se encontró que las personas entienden y explican el suicidio en el marco de una dinámica comunitaria, en la cual identifican factores de riesgo (simbólicos, aspectos relativos a la moral y los valores, el uso del tiempo, lo institucional y la organización comunitaria) y algunos factores protectores (el entorno natural, las redes sociales de apoyo y la tranquilidad del lugar). Los primeros excedieron de manera importante, no solo en número sino en el peso otorgado a los factores protectores, lo cual podría explicar por qué es una comunidad que presenta altas tasas de intento de suicidio y suicidio.

$\underline{3}$ Se refiere a quienes han sufrido la muerte de una persona cercana por suicidio. Esto, independientemente del tipo de vínculo: amistad, familiar, compañero/compañera de trabajo, vecino/vecina, entre muchos otros.

\section{Referencias Bibliográficas}

Abarca Rodríguez, A., Alpízar Rodríguez, F., Sibaja Quesada, G., y Rojas Benavides, C. (2012). Técnicas cualitativas de investigación. San José: Editorial Universidad de Costa Rica.

Carmona Parra, J. A., Torbón Hoyos, F., Jaramillo Estrada, J. C., y Areiza Sánchez, Y. A. (2010). El suicidio en la pubertad y la adolescencia. Un abordaje desde la Psicología Social. Medellín: Fondo Editorial Funlam. 
Chávez Hernández, A. M., Macías García, L. F., y Luna Lara, M. G. (2012). Notas suicidas mexicanas. Un análisis cualitativo. Pensamiento Psicológico, 9(17). En http://www.redalyc.org/pdf/801/80122596003.pdf.

García Alba, J. E., Quintanilla Montoya, R., Sánchez Loyo, L. M., Morfín López, T., y Cruz Gaitán, J. I. (2011). Consenso Cultural sobre el Intento de Suicidio en Adolescentes. Revista Colombiana de Psicología,20(2), 167-179. En http://www.redalyc.org/articulo.oa?id=80421265002

Hernández, R., Fernández, C., y Baptista, P. (2006). Metodología de la Investigación. México: Editorial McGraw Hill Interamericana.

Madelein, L. (2003). Interviews. Interviews. The A-Z of Social Research (pp. 167-172). London: SAGE Publications, Ltd.

Martín Zurro, A., y Jodar, G. (2011). Atención familiar y salud comunitaria. Barcelona: ELSEIVER.

Montero, M. (2004). Introducción a la Psicología Comunitaria. Desarrollo, conceptos y procesos. Buenos Aires: Editorial Paidós.

Rocamora Bonilla, A. (2013). Intervención en crisis en las conductas suicidas. Bilbao: Editorial Desclée de Brouwer, S.A.

Suelves, J. M., y Robert, A. (2012). La conducta suicida: una mirada desde la salud pública. Revista Española de Medicina Legal, 38(4), 137-142. En http://www.elsevier.es/es-revista-revista-espa\%C3\%B1olamedicina-legal-285-articulo-la-conducta-suicida-una-mirada-salud-publica-90167634 